\title{
Treatment of epidermal growth factor receptor inhibitor-induced skin toxicities: do the data support the current practice?
}

\author{
Jonathan Cotliar
}

Received: 1 April 2011 / Accepted: 15 July 2011 / Published online: 4 August 2011

(C) Springer-Verlag 2011

The recognition and treatment of cutaneous toxicities as a consequence of epidermal growth factor receptor inhibitor (EGFRi) use in the treatment of several solid tumors has led to the increasing involvement of dermatologists in the care of cancer patients. Unlike traditional chemotherapeutic agents, where cutaneous complications are more rare, EGFRis frequently induce a variety of skin effects that are both predictable and often severe enough to warrant delaying of treatment or even permanent discontinuation [1]. As a result, it is in the interest of all parties (patients, physicians, drug manufacturers) to devise strategies that will minimize these adverse events to allow for maintenance of cancer therapy. Despite the convergence of such interests, there has been a lack of data based on welldesigned, randomized, and controlled studies from which practitioners can reasonably draw in order to treat these patients when side effects do develop. Though Lacouture et al. have provided a comprehensive and exhaustive review of EGFRi-related toxicities, there continues to be a dearth of validated data concerning management of these treatment complications and a lack of consensus as to how best to approach the majority of patients on EGFRis that will ultimately develop skin toxicities.

In their review, Lacouture et al. allude to the fact that the bulk of interventions used to treat the papulopustular eruption associated with EGFRis are based mostly on case reports or small case series. Of the randomized trials that have been performed, there is conflicting evidence in support of what still remains the standard approach to

\section{J. Cotliar $(\bowtie)$}

Department of Dermatology,

Northwestern University Feinberg School of Medicine,

Chicago, IL, USA

e-mail: j-cotliar@northwestern.edu treatment of this specific reaction, typically a combination of a tetracycline-based oral antibiotic prescribed either prophylactically immediately before EGFRi initiation or shortly thereafter once skin lesions have developed but have not yet reached peak severity. Low- to mediumpotency topical corticosteroids are also commonly used to combat papulopustular lesions when they develop. Jatoi et al. previously concluded that while prophylactic tetracycline does not diminish rash incidence, it seems to ameliorate the severity of papulopustular rash as compared to placebo in addition to improving quality of life of patients on EGFRis [2]. Adding support to the notion of the efficacy of prophylactic tetracycline was the skin toxicity evaluation protocol with panitumumab trial which demonstrated that prophylactic oral doxycycline, emollients, topical corticosteroids, and sunscreen were able to provide a greater than $50 \%$ reduction in the incidence of grade II or greater cutaneous toxicities [3]. These results followed what had been documented 2 years prior with prophylactic oral minocycline allowing for a reduction in the severity of papulopustular eruptions seen in patients on cetuximab during the first month of cancer therapy [4].

Grading of cutaneous toxicities has been problematic, even as the schema have adapted to account for skin eruptions unique to EGFRis and other targeted cancer agents. Jatoi et al. recently concluded that prophylactic tetracycline does not reduce either the incidence or severity of skin eruptions for patients on EGFRis [5]. However, this randomized, double-blinded, and placebo-controlled study employed CTCAE version 3.0, which is fraught with deficiencies when grading EGFRi-induced papulopustular eruptions. In this version, grade I eruptions are differentiated from grade II only on the basis of the presence of symptoms such as pain or pruritus. Furthermore, grade III 
is differentiated from grade II on the basis of either less than $50 \%$ body surface area of involvement (grade II), or greater than $50 \%$ involvement (grade III). No nuance within that version allows for the common presentation of patients on EGFRis with either asymptomatic but diffuse ( $>50 \%$ involvement) eruptions, or those patients with papules and pustules on only a limited amount of skin but with severe pain or pruritus. CTCAE version 4.0 improves upon these shortcomings by using more incremental measurements of percentage of body surface area affected in grades I-III and includes previously ignored features such as interference with ADLs and presence of superinfection. It would be interesting to see if the employment of CTCAE 4.0 and restratification of subjects based on the newer version would be able to detect differences in the number of patients with grades II and III skin eruptions by these new criteria and whether such differences would have any impact on the conclusion of Jatoi's study.

In the short term, it is likely that dermatologists will continue to use oral tetracyclines and topical steroids for patients on EGFRis, unless contraindicated, because of the lack of an established alternative, even with murky data to support their use. It is imperative that oncologists and dermatologists work together to enroll patients in welldesigned trials that use sensible and uniform grading of skin sequelae in order to produce consensus for patients that ideally can maintain their course of cancer treatment with little or no interruption.

Acknowledgments This study was supported by the Irene D. Pritzker Foundation Scholar Award.

Conflict of Interest Jonathan Cotliar is a consultant for Amgen, Inc.

\section{References}

1. Boone SL et al (2007) Impact and management of skin toxicity associated with anti-epidermal growth factor receptor therapy: survey results. Oncology 72(3-4):152-159

2. Jatoi A et al (2008) Tetracycline to prevent epidermal growth factor receptor inhibitor-induced skin rashes: results of a placebocontrolled trial from the North Central Cancer Treatment Group (N03CB). Cancer 113(4):847-853

3. Lacouture ME et al (2010) Skin toxicity evaluation protocol with panitumumab (STEPP), a phase II, open-label, randomized trial evaluating the impact of a pre-emptive skin treatment regimen on skin toxicities and quality of life in patients with metastatic colorectal cancer. J Clin Oncol 28(8):1351-1357

4. Scope A et al (2007) Randomized double-blind trial of prophylactic oral minocycline and topical tazarotene for cetuximab-associated acne-like eruption. J Clin Oncol 25(34):5390-5396

5. Jatoi A et al (2010) Prophylactic tetracycline does not diminish the severity of epidermal growth factor receptor (EGFR) inhibitorinduced rash: results from the North Central Cancer Treatment Group (Supplementary N03CB). Support Care Cancer (in press). Official journal of the Multinational Association of Supportive Care in Cancer 